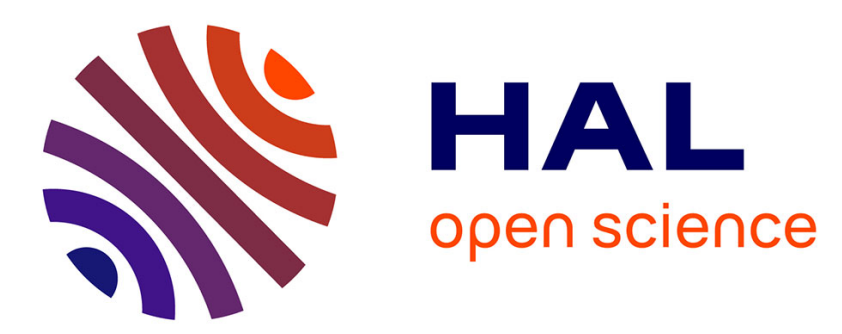

\title{
A well-balanced Runge-Kutta Discontinuous Galerkin method for the Shallow-Water Equations with flooding and drying
}

Alexandre Ern, Serge Piperno, Karim Djadel

\section{- To cite this version:}

Alexandre Ern, Serge Piperno, Karim Djadel. A well-balanced Runge-Kutta Discontinuous Galerkin method for the Shallow-Water Equations with flooding and drying. 2007. hal-00153788

\author{
HAL Id: hal-00153788 \\ https://hal.science/hal-00153788
}

Preprint submitted on 12 Jun 2007

HAL is a multi-disciplinary open access archive for the deposit and dissemination of scientific research documents, whether they are published or not. The documents may come from teaching and research institutions in France or abroad, or from public or private research centers.
L'archive ouverte pluridisciplinaire HAL, est destinée au dépôt et à la diffusion de documents scientifiques de niveau recherche, publiés ou non, émanant des établissements d'enseignement et de recherche français ou étrangers, des laboratoires publics ou privés. 


\title{
A well-balanced Runge-Kutta Discontinuous Galerkin method for the Shallow-Water Equations with flooding and drying
}

\author{
A. Ern, S. Piperno* and K. Djadel \\ CERMICS, École des Ponts, ParisTech, 77455 Marne La Vallée cedex 2, France \\ ern@cermics.enpc.fr, serge.piperno@cermics.enpc.fr
}

\begin{abstract}
SUMMARY
We build and analyze a Runge-Kutta Discontinuous Galerkin method to approximate the one- and two-dimensional Shallow-Water Equations. We introduce a flux modification technique to derive a wellbalanced scheme preserving steady-states at rest with variable bathymetry and a slope modification technique to deal satisfactorily with flooding and drying. Numerical results illustrating the performance of the proposed scheme are presented. Copyright (c) 2000 John Wiley \& Sons, Ltd.
\end{abstract}

KEY WORDS: Runge-Kutta Discontinuous Galerkin; Shallow-Water Equations; source terms; wellbalanced schemes; flooding and drying

\section{Introduction}

Free-surface water flows occur in many domains of practical importance such as coastal and river engineering, dam break problems, or ocean modeling. In many cases, such flows can be satisfactorily modeled by the so-called Shallow-Water Equations (SWE), which are derived by considering the depth-averaged three-dimensional incompressible Navier-Stokes Equations, assuming hydrostatic pressure distribution, and neglecting vertical acceleration and viscous effects $[1,2]$. From a mathematical viewpoint, the SWE are a set of nonlinear first-order partial differential equations of hyperbolic type.

The discretization of the SWE has been the subject of extensive literature. Until recent years, the most commonly chosen numerical methods were Finite Differences (FD), Continuous Finite Elements (CFE) and Finite Volumes (FV). We refer, for instance, to [3] for FD, to $[4,5,6]$ for $\mathrm{CFE}$ and to $[7,8,9,10]$ for $\mathrm{FV}$. The main motivation for using $\mathrm{FV}$ is that such methods are especially tailored to discretize conservation laws possibly with shocks, usually producing approximate solutions with local conservation properties. The main drawback of

*Correspondence to: CERMICS, École des Ponts, ParisTech, 77455 Marne La Vallée cedex 2, France

Contract/grant sponsor: Direction de la Recherche et des Affaires Scientifiques et Techniques (DRAST) of the Ministère de l'équipement, des transports, de l'aménagement, du tourisme et de la mer; contract/grant number: 04 DST 607 
FV is their low order of convergence, even in the case of smooth solutions. To avoid this situation, one can enhance the order of the spatial approximation by using slope reconstruction techniques like the MUSCL scheme (leading to limited orders of convergence on unstructured meshes anyway). Another possibility consists of using higher order polynomials, leading to so-called Discontinuous Galerkin (DG) methods. DG methods approximate the solution in a finite element setting, but in contrast to CFE which use trial and test spaces spanned by continuous piecewise polynomial functions, DG methods use trial and test spaces spanned by piecewise polynomial functions without enforcing explicitly any continuity between adjacent mesh cells. DG methods with polynomial order set to zero can be interpreted as FV schemes.

Since their introduction more than thirty years ago (see [11, 12] for pioneering works), DG methods have experienced a vigorous development. On a given mesh and using a fixed polynomial order, DG methods involve more degrees of freedom than CFE. However, DG methods possess several attractive features, namely they are well-suited to $h p$-adaptive procedures, they can be implemented on arbitrary meshes without enforcing geometric conformity, and they are amenable to parallel computation owing in particular to the blockdiagonal structure of the mass matrix. Moreover, when approximating conservation laws, DG methods lead to local conservation properties at the cell level, as in FV. We refer to $[13,14]$ for a general review of DG methods.

Significant progress in the application of DG methods to the SWE has been achieved in the last few years $[15,16,17,18,19,20,21,22,23,24,25]$. However, two issues relevant in many applications, namely preserving steady-states at rest with variable bathymetry and properly handling flooding and drying, have not been addressed in previous work, with the exception of [17] where a moving mesh was used to deal with dry areas in a one-dimensional setting; the extension to two space dimensions does not seem to be straightforward. The main purpose of this work is to design and analyze a discretization by DG methods of the SWE that can satisfactorily handle the two issues above.

- A desirable feature of discretization schemes for the SWE involving bathymetric terms is to preserve equilibrium states and especially steady-states at rest. However, this property is not satisfied by the usual schemes because it requires a compatibility between the numerical flux and the approximation of the source term. In the framework of FV, several techniques have been proposed to satisfy this property, leading to so-called well-balanced schemes; see $[7,9,10]$ where bathymetric terms are included in the flux calculation and [8] where a so-called upwind discretization of the bathymetric term is proposed. In the present work, we derive a flux modification technique for DG methods inspired from the hydrostatic reconstruction developed for a kinetic scheme in [7].

- Many applications of the SWE involve flooding and drying. One major difficulty when dealing with such processes is to guarantee that the discrete water depth remains nonnegative. Besides their lack of physical meaning, negative values of the water depth lead to difficulties in the computation of the numerical fluxes since the wave speed involves the square root of the water depth. In the present work, we introduce a slope modification technique based on the idea of threshold usually used in the framework of FV. Moreover, we use the HLLE flux [26] in one space dimension and the HLLC [20] flux in two space dimensions which, contrary to Roe's flux for example, ensure a property of nonnegativity for the approximate water depth [27].

This paper is organized as follows. In $\S 2$, the SWE and the main features of the Runge- 
Kutta Discontinuous Galerkin (RKDG) scheme introduced in [20] to approximate the SWE are restated. In $\S 3$, the flux modification technique yielding a well-balanced RKDG method is analyzed. In $\S 4$, the slope modification technique to deal with flooding and drying is described. In $\S 5$, numerical tests are presented to illustrate the performance of the proposed method. Conclusions are reached in $\S 6$. For completeness, an appendix briefly describes the HLLE and the HLLC fluxes.

\section{Approximation of SWE by RKDG methods}

This section restates the main features of the classical RKDG scheme introduced in [20] to approximate the SWE. This scheme will serve as the basis for the new developments presented in $\S 3$ and $\S 4$.

\subsection{Governing Equations}

Let the domain $\Omega$ be an open bounded subset of $\mathbb{R}^{d}, d \in\{1,2\}$, and let $T>0$ be the simulation time. Let $g$ denote the gravitational acceleration and let $b: \Omega \longrightarrow \mathbb{R}$ denote a smooth function representing the bathymetry. Let $\left(x_{1}, \ldots, x_{d}\right)$ denote the spatial coordinates; summation convention for repeated indices is used in the sequel. The SWE can be written as follows:

$$
\left\{\begin{array}{l}
\left.\frac{\partial W}{\partial t}+\frac{\partial \mathbb{F}_{i}(W)}{\partial x_{i}}=\mathbb{S}(W, b) \text { in } \Omega \times\right] 0, T[, \\
\text { Initial and Boundary conditions },
\end{array}\right.
$$

where $W:=(\zeta, q): \Omega \times[0, T] \longrightarrow \mathbb{R}^{m}, m:=d+1$, denotes the conservative variables, $\zeta$ being the (scalar-valued) water depth and $q$ the $\left(\mathbb{R}^{d}\right.$-valued) discharge of the flow with components $\left(q_{1}, q_{2}\right)$ in two space dimensions. Moreover, the source term $\mathbb{S}(W, b)$ and the flux functions $\left\{\mathbb{F}_{i}(W)\right\}_{1 \leq i \leq d}$ are defined for $d=1$ as

$$
\mathbb{S}(W, b):=\left(\begin{array}{c}
0 \\
-g \zeta \frac{\partial b}{\partial x_{1}}
\end{array}\right), \quad \mathbb{F}_{1}(W):=\left(\begin{array}{c}
q \\
\frac{q^{2}}{\zeta}+\frac{g}{2} \zeta^{2}
\end{array}\right),
$$

and for $d=2$ as

$$
\mathbb{S}(W, b):=\left(\begin{array}{c}
0 \\
-g \zeta \frac{\partial b}{\partial x_{1}} \\
-g \zeta \frac{\partial b}{\partial x_{2}}
\end{array}\right), \quad \mathbb{F}_{1}(W):=\left(\begin{array}{c}
q_{1} \\
\frac{q_{1}^{2}}{\zeta}+\frac{g}{2} \zeta^{2} \\
\frac{q_{1} q_{2}}{\zeta}
\end{array}\right), \quad \mathbb{F}_{2}(W):=\left(\begin{array}{c}
q_{2} \\
\frac{q_{1} q_{2}}{\zeta} \\
\frac{q_{2}^{2}}{\zeta}+\frac{g}{2} \zeta^{2}
\end{array}\right) .
$$

\subsection{Space discretization and boundary conditions}

Let $\mathcal{T}_{h}$ be a shape-regular mesh composed of triangular elements. For simplicity, it is assumed that $\mathcal{T}_{h}$ covers $\Omega$ exactly, i.e., $\Omega$ is a polygonal domain in two space dimensions. Let $h:=$ $\max _{K \in \mathcal{T}_{h}} h_{K}$, where $h_{K}$ is the diameter of the element $K \in \mathcal{T}_{h}$ and let $n_{K}=\left(n_{K, 1}, \ldots, n_{K, d}\right)^{t}$ be the unit outward normal of $K$. For $K \in \mathcal{T}_{h}$, a set $\sigma \subset \partial K$ is said to be an interface (resp., a boundary face) of $K$ if there is $K^{\prime} \in \mathcal{T}_{h}$ with $K^{\prime} \neq K$ such that $\sigma=K \cap K^{\prime}$ (resp., if 
$\sigma=K \cap \partial \Omega$ ); $E_{h}^{i}(K)$ (resp., $E_{h}^{\partial}(K)$ ) is then defined as the set of interfaces (resp., boundary faces) of $K$. If $\mathcal{T}_{h}$ does not possess hanging nodes, $E_{h}^{i}(K)$ is simply the set of interior faces of $K$. Set $E_{h}(K)=E_{h}^{i}(K) \cup E_{h}^{\partial}(K)$. For $\sigma \in E_{h}^{i}(K), K \in \mathcal{T}_{h}, K_{\sigma}$ denotes the element of $\mathcal{T}_{h}$ sharing the interface $\sigma$ with $K$, and for $\sigma \in E_{h}(K), n_{K, \sigma}$ denotes the unit outward normal of $K$ on $\sigma$ and $|\sigma|$ the $(d-1)$-dimensional measure of $\sigma$. The space $\mathbb{P}^{p}(K), p \in \mathbb{N}, K \in \mathcal{T}_{h}$, denotes the space of polynomial functions of $d$ variables over $K$ of total degree $p$ at most. The DG space is then defined as $\mathbb{P}_{h}^{p}:=\left\{v: \Omega \rightarrow \mathbb{R}:\left.v\right|_{K} \in \mathbb{P}^{p}(K), \forall K \in \mathcal{T}_{h}\right\}$. Note that a matching condition at interfaces is not enforced on functions in $\mathbb{P}_{h}^{p}$.

For all $K \in \mathcal{T}_{h}$, multiply (1) by $v_{h} \in\left[\mathbb{P}^{p}(K)\right]^{m}$, integrate over $K$, and apply Green's formula. This yields the following (continuous-in-time) space approximation of (1) : Find $W_{h}:=\left(\zeta_{h}, q_{h}\right) \in C^{1}\left([0, T],\left[\mathbb{P}_{h}^{p}\right]^{m}\right)$ such that $\left.\forall t \in\right] 0, T\left[, \forall K \in \mathcal{T}_{h}, \forall v_{h} \in\left[\mathbb{P}^{p}(K)\right]^{m}\right.$,

$$
\left\{\begin{array}{l}
\int_{K} v_{h} \frac{\partial W_{h}}{\partial t}+\int_{\partial K} v_{h} \phi_{K}\left(W_{h}\right)-\int_{K} \frac{\partial v_{h}}{\partial x_{i}} \mathbb{F}_{i}\left(W_{h}\right)=\int_{K} v_{h} \mathbb{S}\left(W_{h}, b\right), \\
\text { Initial condition, }
\end{array}\right.
$$

where $\phi_{K}\left(W_{h}\right)$ is the so-called numerical flux. The numerical flux is evaluated as follows: $\forall K \in \mathcal{T}_{h}, \forall \sigma \in E_{h}(K), \forall x \in \sigma$,

$$
\phi_{K}\left(W_{h}\right)(x)= \begin{cases}\phi_{*}\left(\left.W_{h}\right|_{K}(x),\left.W_{h}\right|_{K_{\sigma}}(x), n_{K, \sigma}\right) & \text { if } \sigma \in E_{h}^{i}(K), \\ \phi_{*}\left(\left.W_{h}\right|_{K}(x), W_{h}^{\partial}(x), n_{K, \sigma}\right) & \text { if } \sigma \in E_{h}^{\partial}(K),\end{cases}
$$

where $\phi_{*}: \mathbb{R}^{m} \times \mathbb{R}^{m} \times \mathbb{R}^{d} \longrightarrow \mathbb{R}^{m}$ is a numerical flux function independent of the mesh cell under consideration and where $W_{h}^{\partial}(x)$ is a fictitious outer state that serves to enforce boundary conditions weakly through the numerical fluxes (see below). The functional $\phi_{*}$ has to verify certain conditions such as conservativity, i.e.,

$$
\forall(X, Y, n) \in \mathbb{R}^{m} \times \mathbb{R}^{m} \times \mathbb{R}^{d}, \quad \phi_{*}(X, Y, n)+\phi_{*}(Y, X,-n)=0,
$$

and consistency, i.e.

$$
\forall(X, n) \in \mathbb{R}^{m} \times \mathbb{R}^{d}, \quad \phi_{*}(X, X, n)=\mathbb{F}_{i}(X) n_{i} .
$$

In this work, $\phi_{*}$ is evaluated using the Harten-Lax-van Leer-Einfeldt (HLLE) flux in one space dimension and the Harten-Lax-van Leer-Contact (HLLC) flux in two space dimensions. The main features of these fluxes are briefly described in the appendix.

The actual expression for $W_{h}^{\partial}(x)$ depends on $\left.W_{h}\right|_{K}(x)$ and on the flow regime where the boundary conditions are enforced. For example, in the case of an inflow boundary face in one space dimension, the speeds of the two Riemann invariants computed using $\left.W_{h}\right|_{K}$ are given by

$$
\lambda_{ \pm}:=\frac{\left.q_{h}\right|_{K}}{\left.\zeta_{h}\right|_{K}} \pm \sqrt{\left.g \zeta_{h}\right|_{K}}
$$

Observe that $\lambda_{+}>0$ at an inflow boundary. If $\lambda_{-}$is also positive, the flow is said to be supercritical and one sets $W_{h}^{\partial}=\left(\zeta^{\partial}, q^{\partial}\right)$, where $\zeta^{\partial}$ and $q^{\partial}$ are prescribed values. If $\lambda_{-}$is negative, the flow is said to be subcritical and one usually imposes either $\zeta$ or $q$. More precisely, the conservation of the outward Riemann invariant is written in the form

$$
\frac{\left.q_{h}\right|_{K}}{\left.\zeta_{h}\right|_{K}}-2 \sqrt{\left.g \zeta_{h}\right|_{K}}=\frac{q^{\partial}}{\zeta^{\partial}}-2 \sqrt{g \zeta^{\partial}} .
$$


If the outer discharge $q^{\partial}$ is prescribed, then (6) permits to obtain an outer water depth $\zeta^{\partial}$ using Newton iterations; if the outer water depth $\zeta^{\partial}$ is prescribed, then (6) immediately yields an outer discharge $q^{\partial}$. For a thorough discussion of boundary conditions for SWE and fictitious outer states, we refer to $[28,4]$.

To write (2) in vector form, a set of basis functions in $\left[\mathbb{P}_{h}^{p}\right]^{m}$ must be selected. To exploit the local character of DG methods, the basis functions have support localized at a single mesh cell. On a given mesh cell, the local basis functions are Legendre polynomials in one space dimension and a particular set of modal basis functions constructed using barycentric coordinates in two space dimensions (see [29] for some properties of these modal basis functions). Let $\vec{W}_{h} \in \mathbb{R}^{N}$ denote the component vector of $W_{h}$ with respect to the basis functions; here, $N$ denotes the total number of degrees of freedom, i.e., the dimension of $\left[\mathbb{P}_{h}^{p}\right]^{m}\left(N=M m \frac{(p+d) !}{d ! p !}\right.$ where $M$ denotes the number of mesh cells). Then, upon inverting the mass matrix, (2) can be recast into the form

$$
\frac{d \vec{W}_{h}}{d t}=\mathcal{H}_{h}\left(\vec{W}_{h}\right)
$$

where $\mathcal{H}_{h}: \mathbb{R}^{N} \rightarrow \mathbb{R}^{N}$. Observe that the mass matrix is block diagonal and hence, easily invertible.

\subsection{Time discretization}

The discretization of the time derivative in (7) is performed in an explicit way. Let $\left(t^{k}\right)_{k \in \mathbb{N}}$ be a sequence of discrete times with $t^{0}=0$. Let $(\Delta t)^{k}=t^{k+1}-t^{k}$ be the $(k+1)$-th time step. To construct an approximation $\vec{W}_{h}^{k}$ of $\vec{W}_{h}$ at the discrete time $t^{k}$, a Runge-Kutta (RK) scheme of order $q$ is used. Given an initial condition $\vec{W}_{h}^{0}$, the scheme consists of the following steps: For $k \in \mathbb{N}$, set $\vec{W}_{h}^{k+1,0}=\vec{W}_{h}^{k}$, then for $i \in\{1, \ldots, q\}$, compute the RK sub-iterates

$$
\vec{W}_{h}^{k+1, i}=\sum_{l=0}^{i-1} c_{i}^{l} \vec{w}_{h i}^{l}, \quad \vec{w}_{h i}^{l}=\vec{W}_{h}^{k+1, l}+\frac{d_{i}^{l}}{c_{i}^{l}}(\Delta t)^{k} \mathcal{H}_{h}\left(\vec{W}_{h}^{k+1, l}\right)
$$

and finally set $\vec{W}_{h}^{k+1}=\vec{W}_{h}^{k+1, q}$. The coefficients $c_{i}^{l}$ and $d_{i}^{l}$ in (8) can be found in [13]. To ensure an equal order of accuracy in space and time, a Runge-Kutta scheme of order $(p+1)$ is used, i.e., $q=p+1$.

The time step is determined adaptively by taking $(\Delta t)^{k}:=\min \left((\Delta t)^{*},(\Delta t)_{\mathrm{cfl}}^{k}\right)$ where $(\Delta t)^{*}$ is a user-defined maximal time step and $(\Delta t)_{\mathrm{cfl}}^{k}$ results from the following CFL condition [13]:

$$
(\Delta t)_{\mathrm{cfl}}^{k}:=\frac{1}{2 p+1} \min _{K \in \mathcal{T}_{h}} \inf _{\substack{x \in \partial K \\ \zeta_{h}^{k}(x)>0}} \frac{h_{K}}{\left(\left|\frac{q_{h}^{k}}{\zeta_{h}^{k}} \cdot n_{K}\right|+\sqrt{g \zeta_{h}^{k}}\right)(x)} .
$$

Here, $W_{h}^{k}=\left(\zeta_{h}^{k}, q_{h}^{k}\right)$ is the function in $\left[\mathbb{P}_{h}^{p}\right]^{m}$ associated with the component vector $\vec{W}_{h}^{k}$.

\subsection{Slope limiting}

It is well-known that in the context of conservation laws, a shock can appear in finite time even if the initial data are smooth. Moreover, high-order methods can yield spurious oscillations 
near a shock. To avoid this situation, slope limiting is necessary. Slope limiting consists of replacing the evaluation of $\vec{W}_{h}^{k+1, i}$ in (8) by

$$
\vec{W}_{h}^{k+1, i}=\Lambda_{i}\left(\sum_{l=0}^{i-1} c_{i}^{l} \vec{w}_{h i}^{l}\right), \quad \vec{w}_{h i}^{l}=\vec{W}_{h}^{k+1, l}+\frac{d_{i}^{l}}{c_{i}^{l}}(\Delta t)^{k} \mathcal{H}_{h}\left(\vec{W}_{h}^{k+1, l}\right),
$$

noticing that the evaluation of $\vec{w}_{h i}^{l}$ is kept unchanged [13]. Here, $\Lambda_{i}: \mathbb{R}^{N} \rightarrow \mathbb{R}^{N}$, $i \in\{1, \ldots, q\}$, are operators that firstly detect shocks and mark cells near shocks and then, on the marked cells, restrict the polynomial order to $p=1$ and reconstruct the slope of the approximation using mean-preserving transformations. In [13], the same operator $\Lambda_{i} \equiv \Lambda$ is used at each RK sub-iterate. Here, this technique is used in one space dimension, but to reduce computational costs in two space dimensions, $\Lambda_{i}$ is the identity for $i<q$ and $\Lambda_{q} \equiv \Lambda$, that is, slope limiting is enforced only on the last RK sub-iterate. Furthermore, following the ideas of [24], slope limiting is applied to the free surface height $(\zeta+b)$ rather than to the water depth $\zeta$. To detect shocks, the criterion proposed in [30] is used. For all $K \in \mathcal{T}_{h}$, define the subset $E_{h}^{-}(K)$ of $E_{h}(K)$ as the inflow interfaces or boundary faces of $K$, namely

$$
E_{h}^{-}(K):=\left\{\sigma \in E_{h}(K): \int_{\sigma} q_{h} \cdot n_{K, \sigma} \leq 0\right\} .
$$

Moreover, setting for all $K \in \mathcal{T}_{h}$ and for all $\sigma \in E_{h}(K)$,

$$
\mathcal{I}_{K, \sigma}:=\frac{\left|\int_{\sigma}\left(\left.\zeta_{h}\right|_{K}-\left.\zeta_{h}\right|_{K_{\sigma}}\right)\right|}{h_{K}^{(p+1) / 2}|\sigma|\left|\left\langle\zeta_{h}\right\rangle_{K}\right|}, \quad \mathcal{I}_{K}:=\sum_{\sigma \in E_{h}^{-}(K)} \mathcal{I}_{K, \sigma}
$$

where $\left\langle\zeta_{h}\right\rangle_{K}$ denotes the mean value of $\zeta_{h}$ over $K$, the criterion is to apply slope limiting on $K$ whenever $\mathcal{I}_{K} \geq 1$.

\section{A well-balanced RKDG scheme with flux modification}

The preservation of equilibrium states is a desirable feature for schemes dealing with the SWE. Among these states, we will consider in particular steady-states at rest. These states are defined by the conditions $\zeta+b \equiv C$ (a constant) and $q \equiv 0$ over the domain. Failure to preserve such states leads to so-called numerical waves; see, e.g., [31] for an example in the framework of FV and $\S 5.2 .1$ for an example with DG methods. Approximation schemes that avoid this situation are termed well-balanced schemes. Examples of well-balanced FV schemes include those designed in $[7,8,9,10]$. Unfortunately, the RKDG scheme defined in $\S 2$ is not well-balanced. Indeed, well-balancing requires a compatibility between the numerical flux and the discretization of the source term. The goal of this section is to design a well-balanced RKDG scheme.

A first observation is that it is not possible to obtain $\zeta_{h}+b \equiv C$ simply because $b \notin \mathbb{P}_{h}^{p}$. Hence, we seek for the optimal $\zeta_{h} \in \mathbb{P}_{h}^{p}$ in the least-squares sense, that is, we seek for $\zeta_{h} \in \mathbb{P}_{h}^{p}$ such that $\zeta_{h}+b_{h} \equiv C$ where $b_{h} \in \mathbb{P}_{h}^{p}$ is the $L^{2}$-projection of $b$ onto $\mathbb{P}_{h}^{p}$. Recall that this projection verifies

$$
\int_{K} b v_{h}=\int_{K} b_{h} v_{h}, \quad \forall v_{h} \in \mathbb{P}^{p}(K), \quad \forall K \in \mathcal{T}_{h} .
$$


The well-balanced RKDG scheme with slope limiting is obtained by modifying (2) as follows:

$$
\begin{aligned}
\int_{K} v_{h} \frac{\partial W_{h}}{\partial t} & +\int_{\partial K} v_{h} \phi_{K}\left(W_{h}^{\diamond}\right)-\int_{K} \frac{\partial v_{h}}{\partial x_{i}} \mathbb{F}_{i}\left(W_{h}\right) \\
& =\int_{K} v_{h} \mathbb{S}\left(W_{h}, b_{h}\right)+\int_{\partial K} v_{h} \delta_{K}\left(W_{h}, b_{h}\right) .
\end{aligned}
$$

Here, $W_{h}^{\diamond}:=\left(\zeta_{h}^{\diamond}, q_{h}^{\diamond}\right)$ with for $K \in \mathcal{T}_{h}$,

$$
\left.\zeta_{h}^{\diamond}\right|_{K}:= \begin{cases}\max \left(0,\left.\zeta_{h}\right|_{K}-\max \left(\left.b_{h}\right|_{K_{\sigma}}-\left.b_{h}\right|_{K}, 0\right)\right), & \sigma \in E_{h}^{i}(K), \\ \left.\zeta_{h}\right|_{K}, & \sigma \in E_{h}^{\partial}(K),\end{cases}
$$

while $q_{h}^{\diamond}:=\zeta_{h}^{\diamond} q_{h} / \zeta_{h}$, and where

$$
\delta_{K}\left(W_{h}, b_{h}\right):=\left(\begin{array}{c}
0 \\
\frac{g}{2}\left(\left.\zeta_{h}^{\diamond}\right|_{K} ^{2}-\left.\zeta_{h}\right|_{K} ^{2}\right) n_{K}
\end{array}\right) .
$$

The difference between (2) and (11) is on the one hand that in (11) the numerical flux is evaluated using $W_{h}^{\diamond}$ instead of $W_{h}$ (still using (3)), and on the other hand that the source term consists of a volume contribution $\int_{K} v_{h} \mathbb{S}\left(W_{h}, b_{h}\right)$ (evaluated using the projected bathymetry $\left.b_{h}\right)$ and a surface contribution $\int_{\partial K} v_{h} \delta_{K}\left(W_{h}, b_{h}\right)$. In vector form, (11) can be recast into the form

$$
\frac{d \vec{W}_{h}}{d t}=\mathcal{H}_{h}^{\mathrm{wb}}\left(\vec{W}_{h}\right)
$$

where $\mathcal{H}_{h}^{\text {wb }}: \mathbb{R}^{N} \rightarrow \mathbb{R}^{N}$. The well-balanced RKDG scheme consists of replacing (10) by

$$
\vec{W}_{h}^{k+1, i}=\Lambda_{i}\left(\sum_{l=0}^{i-1} c_{i}^{l} \vec{w}_{h i}^{l}\right), \quad \vec{w}_{h i}^{l}=\vec{W}_{h}^{k+1, l}+\frac{d_{i}^{l}}{c_{i}^{l}}(\Delta t)^{k} \mathcal{H}_{h}^{\mathrm{wb}}\left(\vec{W}_{h}^{k+1, l}\right) .
$$

The key property of the above scheme is given in the following

Proposition 1. The scheme (13) preserves steady-states at rest, i.e., for all $k \in \mathbb{N}$,

$$
\left(\zeta_{h}^{k}+b_{h} \equiv C \text { and } q_{h}^{k} \equiv 0\right) \Rightarrow\left(\zeta_{h}^{k+1}+b_{h} \equiv C \text { and } q_{h}^{k+1} \equiv 0\right),
$$

where $C$ denotes a fixed positive constant.

Proof. Let $W_{h}=\left(\zeta_{h}, q_{h}\right) \in\left[\mathbb{P}_{h}^{p}\right]^{m}$ such that $\zeta_{h}+b_{h} \equiv C$ and $q_{h}=0$ (the superscript $k$ is omitted for brevity). We set $d=2$ for the following of the proof, the case $d=1$ is treated in a similar way. It is clear that it is sufficient to prove that for all $K \in \mathcal{T}_{h}$ and for all $v_{h} \in\left[\mathbb{P}^{p}(K)\right]^{m}$,

$$
\int_{\partial K} v_{h} \phi_{K}\left(W_{h}^{\diamond}\right)-\int_{K} \frac{\partial v_{h}}{\partial x_{i}} \mathbb{F}_{i}\left(W_{h}\right)=\int_{K} v_{h} \mathbb{S}\left(W_{h}, b_{h}\right)+\int_{\partial K} v_{h} \delta_{K}\left(W_{h}, b_{h}\right) .
$$

Since $W_{h}$ corresponds to a steady-state at rest, it is readily verified that

- for all $K \in \mathcal{T}_{h}$ and for all $\sigma \in E_{h}^{i}(K), \zeta_{h}^{\diamond}$ is single-valued on $\sigma$ and equal to $C-\max \left(\left.b_{h}\right|_{K},\left.b_{h}\right|_{K_{\sigma}}\right)$;

- $q_{h}^{\diamond}=0$. 
Using the consistency of the flux function $\phi_{*}$ (see (5)) then yields that

Moreover,

$$
\phi_{K}\left(W_{h}^{\diamond}\right)=\left(\begin{array}{c}
0 \\
\frac{g}{2}\left(\left.\zeta_{h}^{\diamond}\right|_{K}\right)^{2} n_{K, 1} \\
\frac{g}{2}\left(\left.\zeta_{h}^{\diamond}\right|_{K}\right)^{2} n_{K, 2}
\end{array}\right) .
$$

$$
\mathbb{F}_{1}(W):=\left(\begin{array}{c}
0 \\
\frac{g}{2} \zeta_{h}^{2} \\
0
\end{array}\right), \quad \mathbb{F}_{2}(W):=\left(\begin{array}{c}
0 \\
0 \\
\frac{g}{2} \zeta_{h}^{2}
\end{array}\right), \quad \mathbb{S}\left(W_{h}, b_{h}\right):=\left(\begin{array}{c}
0 \\
\frac{g}{2} \frac{\partial \zeta_{h}^{2}}{\partial x_{1}} \\
\frac{g}{2} \frac{\partial \zeta_{h}^{2}}{\partial x_{2}}
\end{array}\right),
$$

where we have used that $\zeta_{h}+b_{h} \equiv C$ to simplify the expression for $\mathbb{S}\left(W_{h}, b_{h}\right)$. Using (12) and Green's formula yields the desired result.

Remark 1. The only property required on the numerical flux for the above result to hold is consistency (but not conservativity).

It is important to assess the accuracy of the above flux modification technique. This motivates the following

Proposition 2. Let $W_{h} \in\left[\mathbb{P}_{h}^{p}\right]^{m}$. Assume that for all $K \in \mathcal{T}_{h},\left.\zeta_{h}\right|_{K}$ is positive and that $\left.\zeta_{h}\right|_{K}$ and $\left.\left(q_{h} / \zeta_{h}\right)\right|_{K}$ are uniformly bounded. Assume that the bathymetry is smooth enough. Then, for all $K \in \mathcal{T}_{h}$, for all $\sigma \in E_{h}^{i}(K)$, and for all $x \in \sigma$,

$$
\left\|W_{h}(x)-W_{h}^{\diamond}(x)\right\|_{\mathbb{R}^{m}}+\left\|\delta_{K}\left(W_{h}, b_{h}\right)\right\|_{\mathbb{R}^{m}} \leq c h_{K}^{p+1},
$$

where $c$ is independent of $\mathcal{T}_{h}$ and where $\|\cdot\|_{\mathbb{R}^{m}}$ denotes any norm on $\mathbb{R}^{m}$.

Proof. Since the bathymetry is smooth enough, classical approximation results imply that for all $K \in \mathcal{T}_{h}$, for all $\sigma \in E_{h}^{i}(K)$, and for all $x \in \sigma$,

$$
\left|b_{h}\right|_{K}(x)-\left.b_{h}\right|_{K_{\sigma}}(x) \mid \leq c h_{K}^{p+1},
$$

whence the conclusion is readily inferred.

Proposition 2 shows that the flux modification technique induces a perturbation of the original RKDG scheme of order $h^{p+1}$. Since the problem is nonlinear, it cannot be inferred that the error induced by this perturbation is necessarily of the same order. Numerical results reported in $\S 5$ confirm that the present flux modification technique preserves the high-order accuracy of the RKDG method.

\section{Slope modification for flooding and drying}

When the problem involves flooding and drying, it is necessary to prevent the discrete water depth from taking negative values. To this purpose, a procedure, similar in spirit to slope limiting, is introduced. On each mesh cell where the minimum (computed over the integration points) of $\zeta_{h}$ is lower than a threshold $\varepsilon$, the following steps are taken.

For $p=0$, the procedure is similar to that used in FV, namely setting to zero $\zeta_{h}$ and $q_{h}$. For $p \geq 2$, the discrete solution is first projected onto linears and then the procedure for $p=1$ is applied elementwise as follows: 
- If the average of $\zeta_{h}$ is negative, then $\zeta_{h}$ and $q_{h}$ are set to zero.

- If the average of $\zeta_{h}$ is nonnegative, this value is kept but the gradient of $\zeta_{h}$ is modified in such a way that $\zeta_{h}$ vanishes at vertices with negative value. More specifically in two space dimensions, let $K$ be the reference triangle with vertices $v_{0}:=(0,0), v_{1}:=(1,0)$ and $v_{2}:=(0,1)$. Introduce the nodal polynomial basis functions $p_{0}:=1-x-y, p_{1}:=x$ and $p_{2}:=y$. Let $\zeta_{h} \in \mathbb{P}^{1}(K)$ be such that $\zeta_{h}:=\sum_{j=0}^{2} \zeta_{j} p_{j}$ and assume that $\zeta_{h}$ has negative values on $K$. Let $\left\langle\zeta_{h}\right\rangle_{K}$ denote the mean of $\zeta_{h}$ over $K$. If $\zeta_{h}$ is negative at only one vertex, say $v_{i}$ with $i \in\{0,1,2\}$, then

$$
\zeta_{h}^{\prime}:=\frac{\left\langle\zeta_{h}\right\rangle_{K}}{\left\langle\zeta_{h}\right\rangle_{K}-\zeta_{i}}\left(\zeta_{h}-\zeta_{i}\right)
$$

If $\zeta_{h}$ is negative at two vertices, say $v_{i_{1}}$ and $v_{i_{2}}$ with $i_{1}, i_{2} \in\{0,1,2\}$, then

$$
\zeta_{h}^{\prime}:=\frac{\left\langle\zeta_{h}\right\rangle_{K}}{\left\langle p_{i}\right\rangle_{K}} p_{i},
$$

where $i \in\{0,1,2\} \backslash\left\{i_{1}, i_{2}\right\}$. It is straightforward to verify that

$$
\begin{aligned}
& \left\langle\zeta_{h}^{\prime}\right\rangle_{K}=\left\langle\zeta_{h}\right\rangle_{K}, \\
& \forall x \in K, \quad \zeta_{h}^{\prime}(x) \geq 0 .
\end{aligned}
$$

Finally, the discharge $q_{h}$ is modified by only setting its value to zero at those vertices where $\zeta_{h}$ has been modified. Moreover, $q_{h}$ is also modified using a similar procedure whenever the discrete velocity norm is larger than a prescribed upper bound for the velocity in the flow. The overall transformation preserves mass because of (14) (as long as the average of $\zeta_{h}$ is nonnegative), but not discharge.

The well-balanced RKDG scheme with slope modification consists of replacing (13) by

$$
\vec{W}_{h}^{k+1, i}=\Lambda_{i}\left(\sum_{l=0}^{i-1} c_{i}^{l} \vec{w}_{h i}^{l}\right), \quad \vec{w}_{h i}^{l}=\vec{W}_{h}^{k+1, l}+\frac{d_{i}^{l}}{c_{i}^{l}}(\Delta t)^{k} \mathcal{H}_{h}^{\mathrm{wb}}\left(\Upsilon \vec{W}_{h}^{k+1, l}\right),
$$

where $\Upsilon: \mathbb{R}^{N} \rightarrow \mathbb{R}^{N}$ is the mean-preserving and nonnegativity-enforcing transformation defined above. Slope limiting is not applied at the same time as slope modification, since the latter can activate artificially the former.

\section{Numerical tests}

Test cases presented in this section are regrouped into three subsections. In $\S 5.1$, we illustrate the ability of the classical RKDG scheme described in $\S 2$ to approximate smooth solutions with high accuracy and to capture sharply shocks for constant bathymetry. In $\S 5.2$, we illustrate the fact that the well-balanced RKDG scheme designed in $\S 3$ performs equally well in terms of accuracy and shock capturing when the bathymetry is variable. In $\S 5.3$, we assess the slope modification technique designed in $\S 4$ to handle flooding and drying within the well-balanced RKDG scheme. In the sequel, we set $g=9.81 \mathrm{~m} / \mathrm{s}^{2}$. When evaluating convergence rates below, the parameter $h$ representative of a given triangulation is evaluated as the maximal length of an edge in the triangulation. Let us mention that the unstructured meshes considered henceforth are quite regular and smooth (in general, the minimal length of an edge in the mesh is larger than $h / 3)$. 


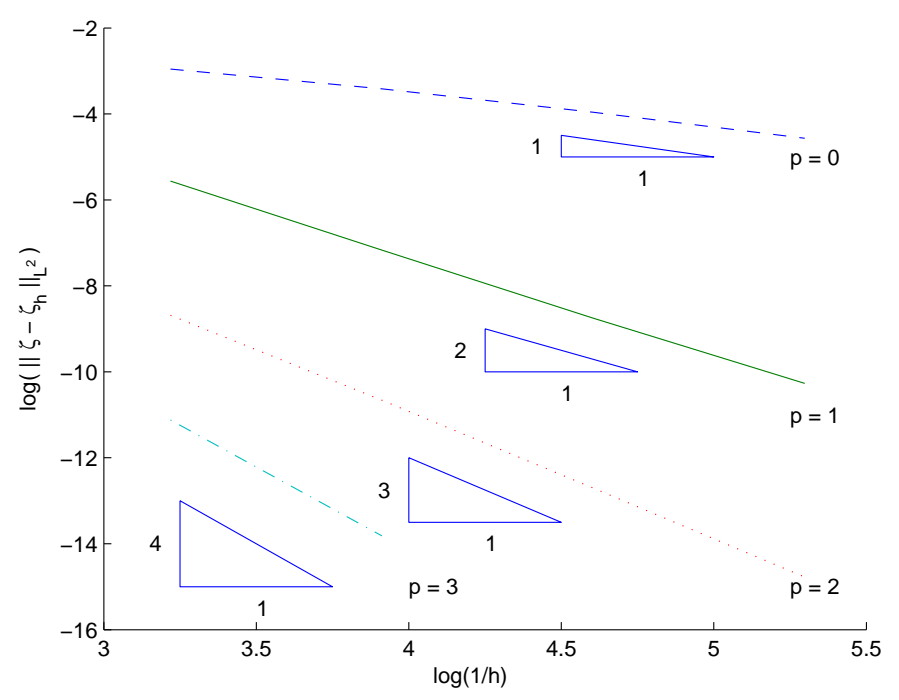

Figure 1. Test case with smooth solution: $L^{2}$-error on the water depth for $p \in\{0,1,2,3\}$.

\subsection{Constant bathymetry}

5.1.1. Smooth solutions Consider a one-dimensional domain $\Omega$ of length $10 \mathrm{~m}$ and a final simulation time of $T=0.5 \mathrm{~s}$. The initial datum is $\zeta_{0}=\left(1+0.2 e^{-100(x-0.5)^{2}}\right) \mathrm{m}$ and $q_{0}=0 \mathrm{~m}^{2} / \mathrm{s}$. Since an analytical solution is not available, the error is calculated with respect to a reference solution computed on an uniform mesh of 200 cells with polynomial degree $p=3$. Figure 1 presents the $L^{2}$-error on the water depth for various mesh sizes. In all cases, the convergence rate is $(p+1)$ as expected.

5.1.2. Oblique hydraulic jump The aim of this test case is to study the performance of the classical RKDG scheme in the case where the exact solution presents a shock. We consider the standard test case of an oblique hydraulic jump on a flat bottom [32]. The definition of the problem is illustrated in Figure 2: a uniform horizontal inflow (state $\left(\zeta_{u}, q_{1 u}, q_{2 u}\right)$ ) is deflected by an oblique wall with deflection angle $\alpha$. The steady analytical solution presents an oblique jump (angle $\beta$ with the horizontal axis) separating the inflow zone from a constant downstream state $\left(\zeta_{d}, q_{1 d}, q_{2 d}\right)$ with $\left(q_{1 u}, q_{2 d}\right)=q_{d}(\cos (\alpha), \sin (\alpha))$. The Rankine-Hugoniot jump relations yield:

$$
q_{1 u}^{2} \sin ^{2} \beta=q_{d}^{2} \sin ^{2}(\beta-\alpha)=g \zeta_{u} \zeta_{d} \frac{\zeta_{u}+\zeta_{d}}{2}, \quad \tan (\alpha)=\frac{\left(\zeta_{d}-\zeta_{u}\right) \sin \beta}{\zeta_{u} \sin ^{2} \beta+\zeta_{d} \cos ^{2} \beta} .
$$

Imposing $\zeta_{u}=1 \mathrm{~m}, \zeta_{d}=1.5 \mathrm{~m}$ and $\beta=30^{\circ}$ yields the approximate values: $\alpha \approx 8.9483^{\circ}$, $q_{1 u} \approx 8.5776 \mathrm{~m}^{2} / \mathrm{s},\left(q_{1 d}, q_{2 d}\right) \approx(11.7941,1.8571) \mathrm{m}^{2} / \mathrm{s}$. Furthermore, the initial condition is $\zeta_{0}=1 \mathrm{~m}$ and $q_{0}=(8.57,0) \mathrm{m}^{2} / \mathrm{s}$. We compute the DG solution on unstructured meshes for the degree of approximation $p=1$. The initial and final approximations are represented in Figure 3. For all the conserved variables, the convergence rate of the $L^{2}$-error is $\frac{1}{2}$ as expected 


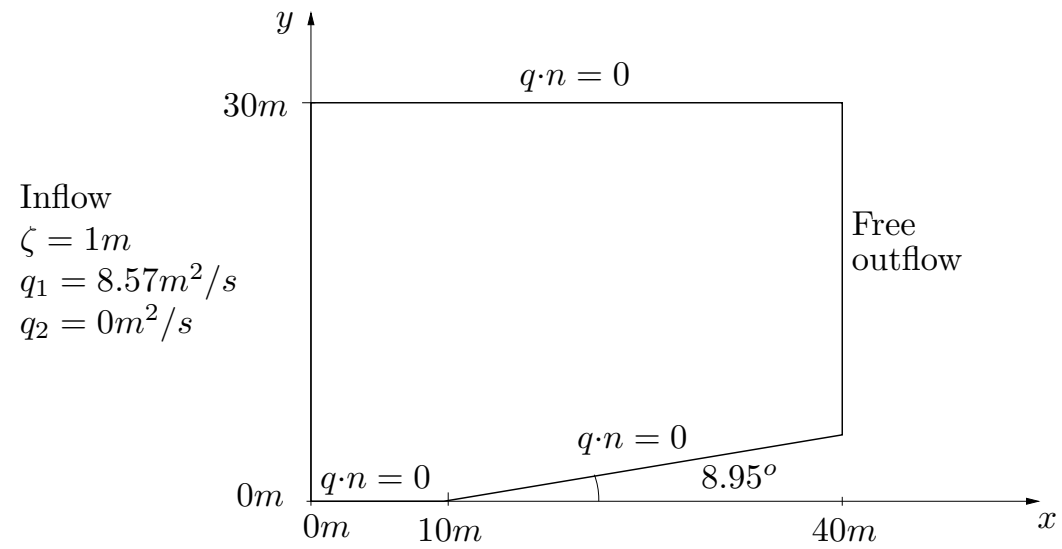

Figure 2. Oblique hydraulic jump: problem setting.

owing to the presence of a shock and the use of unfitted meshes (i.e., the oblique shock crosses some mesh cells).
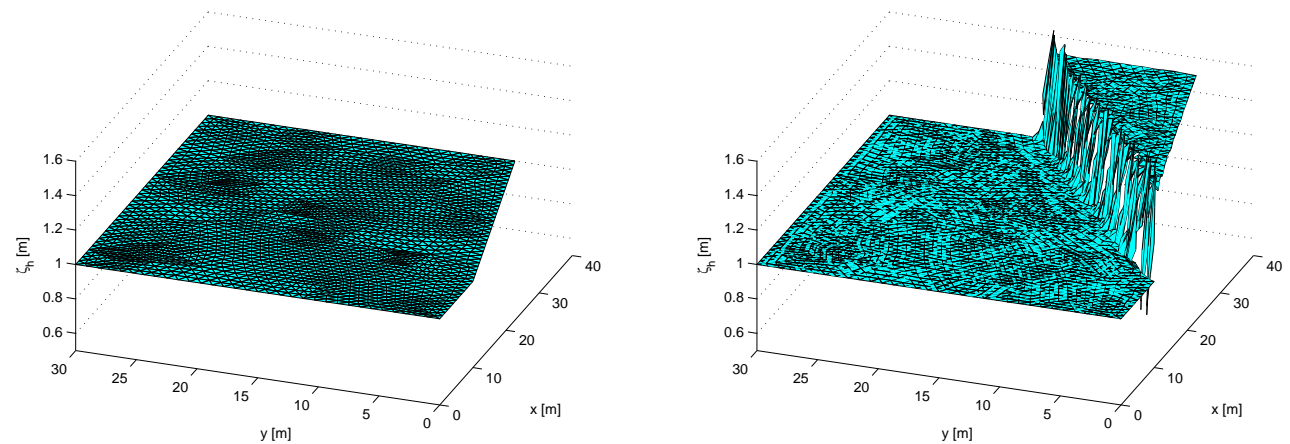

Figure 3. Oblique hydraulic jump: initial (left) and final (right) approximate water heights for $p=1$.

\subsection{Variable bathymetry}

5.2.1. Steady-state at rest The preservation of steady-states at rest by the well-balanced RKDG scheme can be illustrated on one-dimensional setting. The initial condition is $\zeta_{0}+b=$ $1 m$ and $q_{0}=(0,0) m^{2} / s$ with $b(x)=\left(10 e^{-x^{2}}+15 e^{-(x-2.5)^{2}}+10 e^{-(x-5)^{2} / 2}+6 e^{-2(x-7.5)^{2}}+\right.$ $\left.16 e^{-(x-10)^{2}}\right) / 20$. Figure 4 presents the approximate solution at time $T=1 s$ obtained by the classical RKDG scheme and by the well-balanced RKDG scheme for $p=2$ and an uniform step size of $h=1 \mathrm{~m}$. The importance of numerical waves introduced by the classical scheme and their elimination by the flux modification technique are clearly illustrated. 

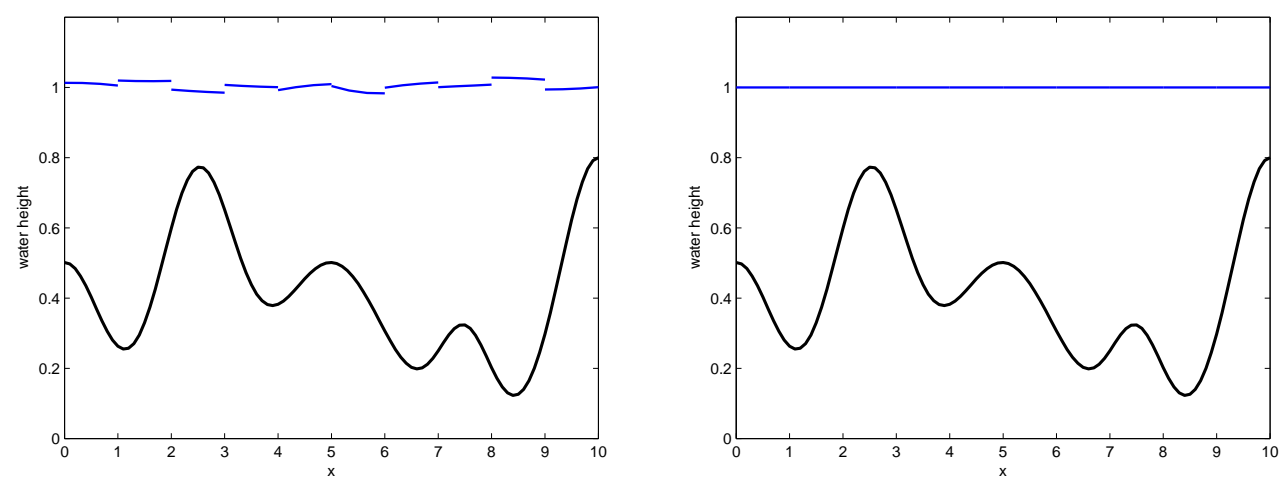

Figure 4. Steady-state at rest: water height for the classical RKDG scheme (left) and for the wellbalanced RKDG scheme (right) for $p=2$ at time $T=1 s$ (bathymetry in bold line).

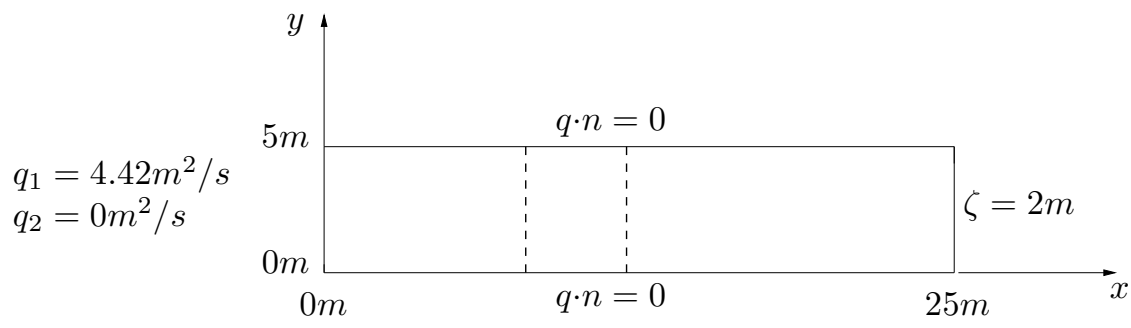

Figure 5. Subcritical flow: problem setting (the leading and trailing edges of the bump are indicated by a dashed line).

5.2.2. Subcritical flow To assess the order of accuracy of the scheme, we now consider a classical test case of a subcritical flow over a bump [33]. The definition of the problem is illustrated in Figure 5. The bathymetry is $b(x, y)=\max \left(0,0.2-0.05(x-10)^{2}\right)$ and the initial condition $\zeta_{0}+b=2 m$ and $q_{0}=(0,0) \mathrm{m}^{2} / \mathrm{s}$. After a finite time (for this test, we set $T=600 \mathrm{~s}$ ), the solution reaches a steady-state (see Figure 6). Using structured, fitted meshes in which the lines of discontinuity of the slope of the bathymetry coincide with mesh cell interfaces, the optimal order of convergence $(p+1)$ of the RKDG method is recovered. The errors in the $L^{2}$-norm on the water depth for $p \in\{0,1,2\}$ are plotted in the left part of Figure 7 . Using unstructured, unfitted meshes, the optimal order of convergence $(p+1)$ of the RKDG method is not preserved. The errors in the $L^{2}$-norm on the water depth for $p \in\{0,1,2\}$ are plotted in the right part of Figure 7 . The maximum order of convergence is $\frac{3}{2}$; this can be explained by the fact that the exact solution is continuous but not $C^{1}$ inside some mesh elements.

5.2.3. Transcritical flow with shock We consider the same domain and bathymetry as in the previous test case but the initial condition is $\zeta_{0}+b=0.33 \mathrm{~m}$ and $q_{0}=(0,0) \mathrm{m}^{2} / \mathrm{s}$. Moreover, the inflow discharge and the outflow water depth are $q_{\text {in }}=(0.18,0) \mathrm{m}^{2} / \mathrm{s}$ and $h_{\text {out }}=0.33 \mathrm{~m}$ [33]. The obtained steady-state (observed at time $T=100 \mathrm{~s}$ ) presents a stationary shock (see Figure 8). The errors in the $L^{2}$-norm on all the conservative variables (water depth and 

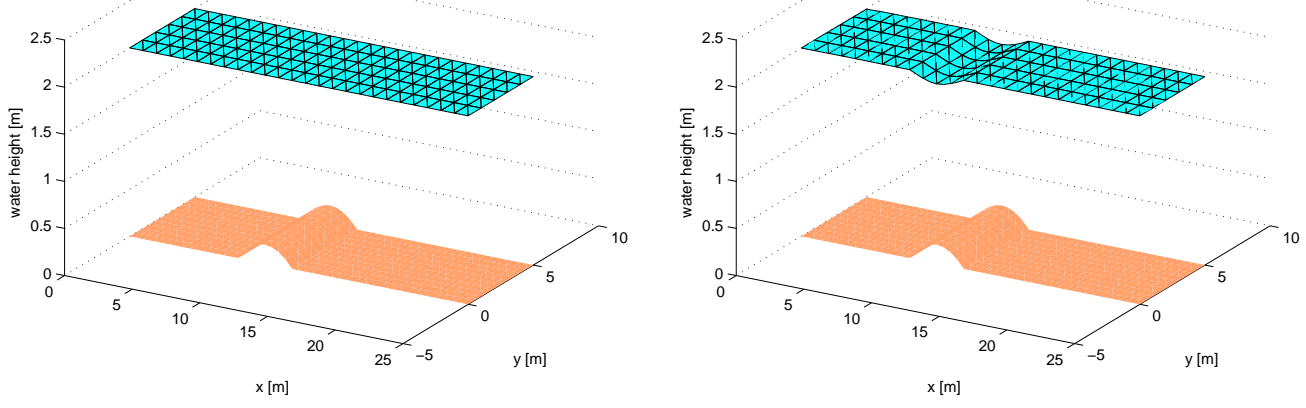

Figure 6. Subcritical flow: initial (left) and final (right) approximate water heights for $p=1$ (structured, fitted meshes).
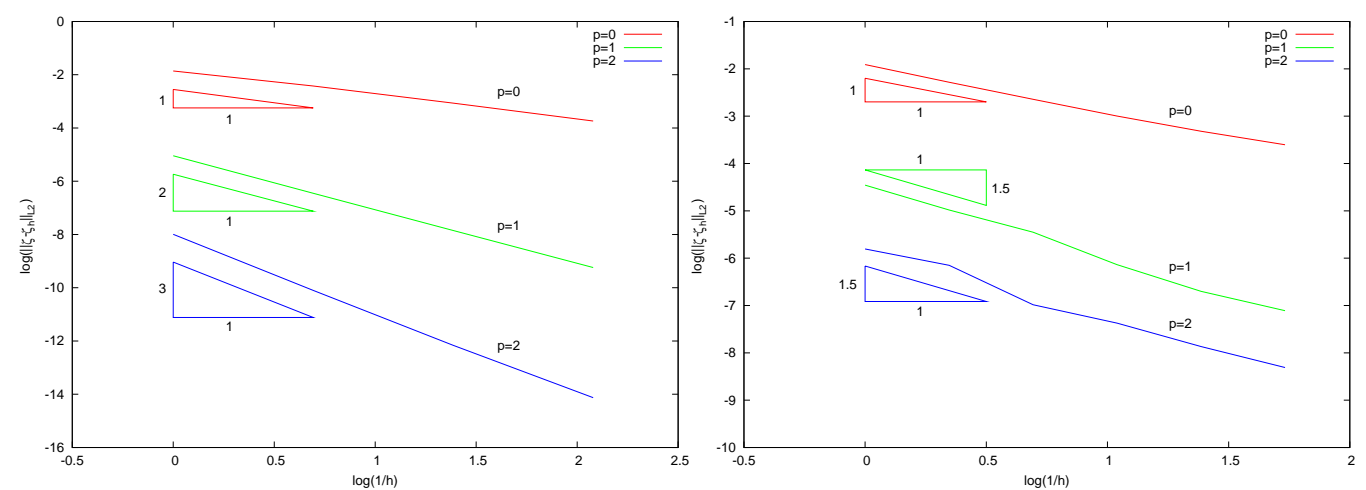

Figure 7. Subcritical flow: $L^{2}$-error on the water depth for $p \in\{0,1,2\}$ : structured, fitted meshes (left) and unstructured, unfitted meshes (right).

discharge) are illustrated in Figure 9 for $p=1$. As for reconstructed FV methods [7], the observed order of convergence is $\frac{1}{2}$.

\subsection{Flooding and drying}

5.3.1. Ritter solution [34] We now study the capacity of the slope modification technique to treat flooding. The domain $\Omega$ is a $50 \mathrm{~m} \times 40 \mathrm{~m}$ rectangle and the bottom is flat. The initial discharge is $q=(0,0) \mathrm{m}^{2} / \mathrm{s}$ and the initial water depth is set to zero for $x>20 \mathrm{~m}$ and to $\zeta_{0}$ for $x<20 \mathrm{~m}$. The analytical solution is self-similar, i.e., it depends only on $\xi=\frac{x-20}{t}$. It is given by

$$
\begin{cases}\text { if } \xi<-\sqrt{g \zeta_{0}}: & \zeta(x, t)=\zeta_{0}, q(x, t)=0 \\ \text { else if } \xi>2 \sqrt{g \zeta_{0}}: & \zeta(x, t)=0, q(x, t)=0 \\ \text { else } & \zeta(x, t)=\frac{1}{9 g}\left(\xi-2 \sqrt{g \zeta_{0}}\right)^{2}, u(x, t)=\frac{2}{3}\left(\xi+\sqrt{g \zeta_{0}}\right)\end{cases}
$$



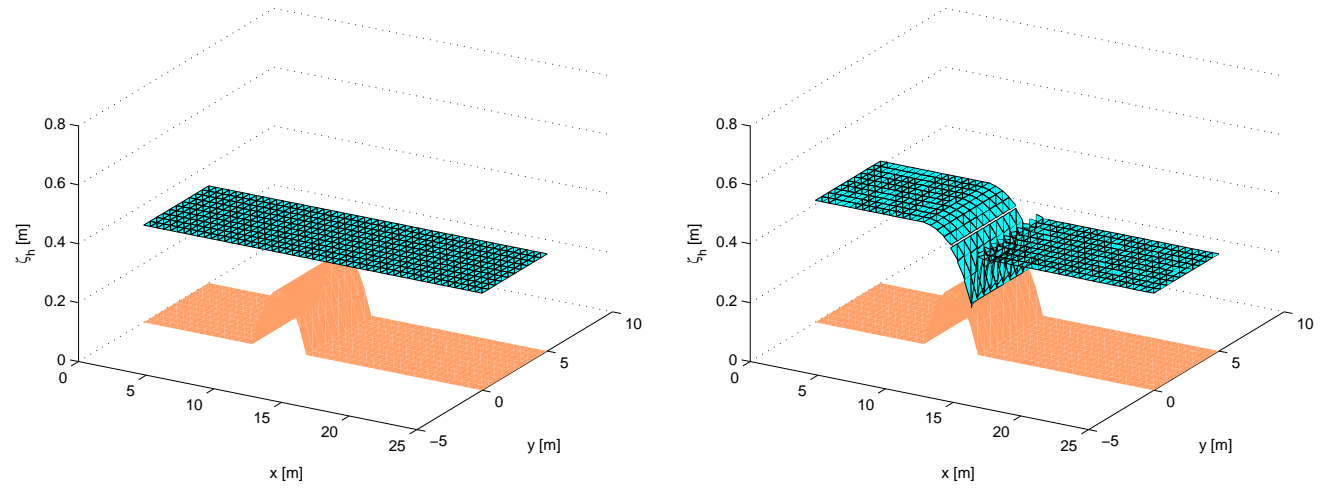

Figure 8. Transcritical flow with shock: initial (left) and final (right) approximate water heights for $p=1$.

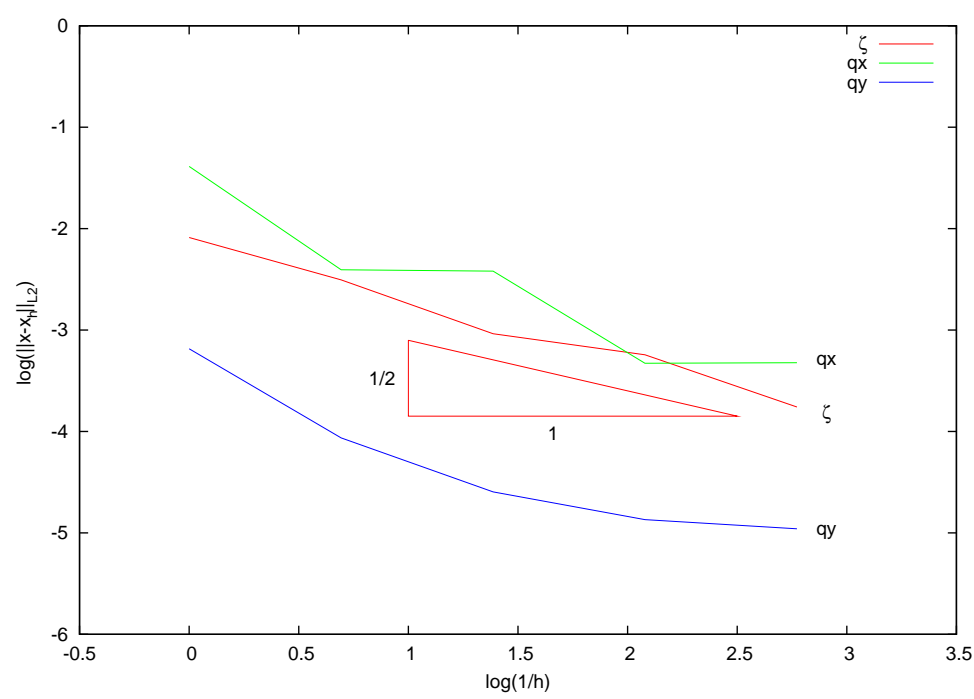

Figure 9. Transcritical flow with shock: $L^{2}$-errors on all the conservative variables $(p=1)$.

We have taken $\zeta_{0}$ such that $g \zeta_{0}=1 \mathrm{~m}^{2} / \mathrm{s}^{2}$. The simulation time is $T=10 \mathrm{~s}$ (such that the rarefaction wave does not reach the boundary of the domain) and we consider unstructured meshes (however, meshes are fitted to the discontinuity in the initial solution). The threshold $\varepsilon$ introduced in Section 4 for the slope modification technique is set to $10^{-6}$. The initial and final approximate water depths are plotted for $p=1$ in Figure 10. The test case is solved starting with the analytical solution at time $t=2$. Thus the solution is at least everywhere continuous, but not continuously differentiable. The limiting process is not used since the solution is smooth enough. The errors in the $L^{2}$-norm on the water depth are presented in Figure 11 for $p \in\{0,1,2\}$. The error on the water depth behaves like $h^{0.8}$ for $p=0$ (an 

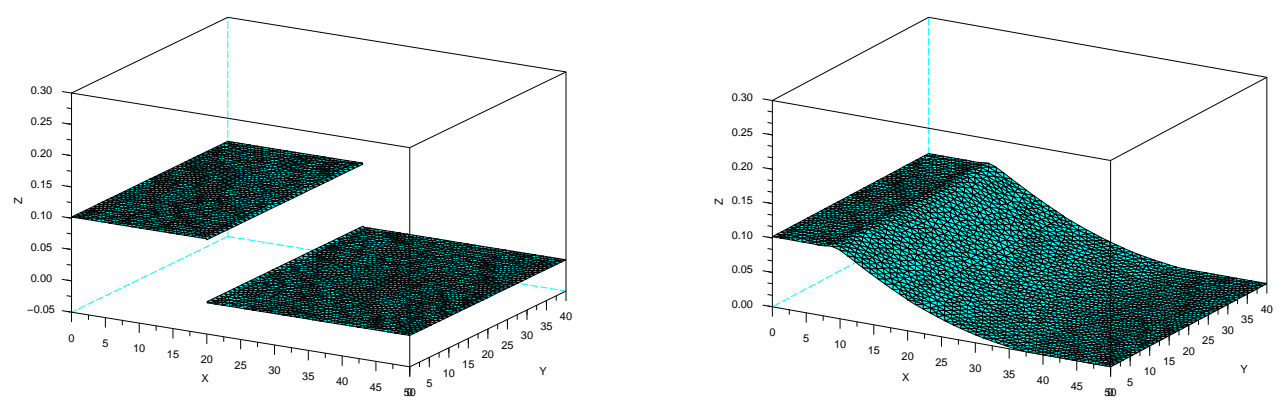

Figure 10. Rarefaction wave: initial (left) and final (right) approximate water depths for $p=1$.

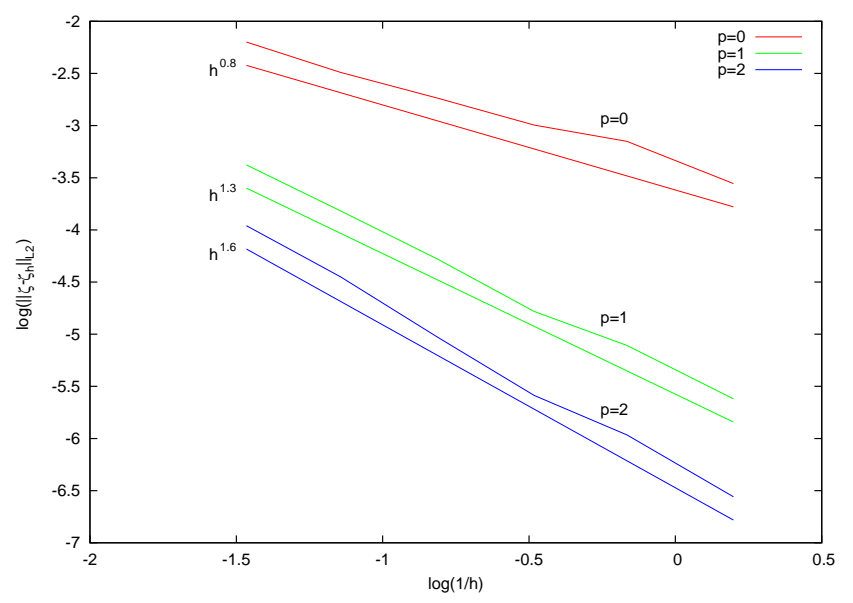

Figure 11. Rarefaction wave: overall $L^{2}$-error on the water depth for $p \in\{0,1,2\}$.

order of convergence between $1 / 2$ and 1 is expected), and respectively like $h^{1.3}$ and $h^{1.6}$ for $p=1$ and $p=2$ (since the solution is not smooth at the left end of the rarefaction wave, the global accuracy should be limited to $\frac{3}{2}$ ). It is interesting to notice that the error is localized in the regions where the solution is not very smooth (near both ends of the rarefaction fan), which means that the accuracy of the method in the present case is preserved far from relative singularities. This is shown by Figure 12 which represents the $L^{2}$-norm of the error on the water depth in the region $\{x \in[15 ; 35]\}$ at time $t=10 s$ for $p \in\{0,1,2\}$. One finds numerically that these errors behave respectively for $p \in\{0,1,2\}$ like $h^{0.8}, h^{2.5}$, and $h^{3.2}$.

5.3.2. Parabolic bowl The aim is to assess the capacity of the method to treat flooding and drying. We consider a parabolic bowl (the bottom corresponds to a paraboloid of revolution, i.e., $b(x, y)=\alpha r^{2}$ with $r^{2}=x^{2}+y^{2}$ and $\alpha$ is a positive constant) for which the exact solution has a periodic behavior and the free surface is an oscillating paraboloid of revolution. The analytical solution (see [35] for more details) is such that $\zeta(r, t)$ is non-zero for $r<\sqrt{\frac{X+Y \cos \omega t}{\alpha\left(X^{2}-Y^{2}\right)}}$ 


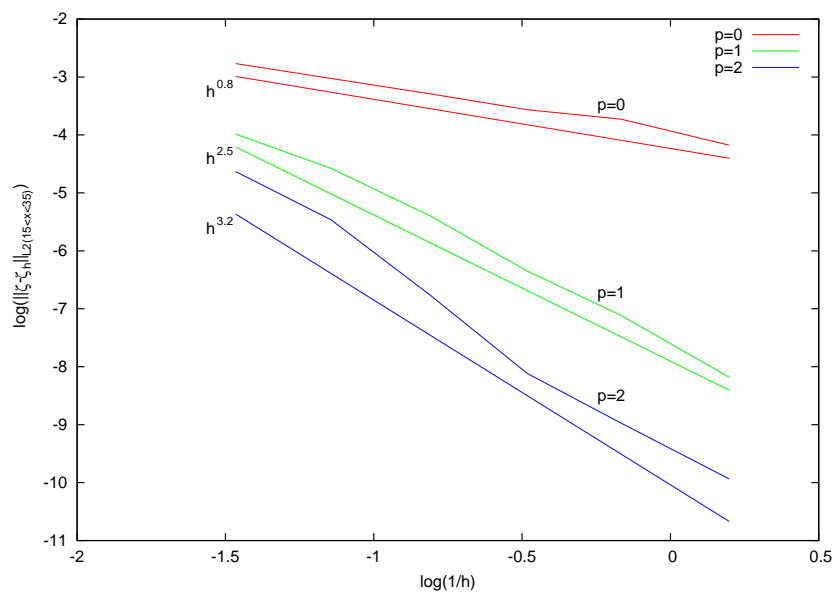

Figure 12. Rarefaction wave: local $L^{2}$-error over $\{x \in[15 ; 35]\}$ on the water depth for $p \in\{0,1,2\}$.

(with $\omega^{2}=8 g \alpha, X$ and $Y$ are constants such that $X>0$ and $|Y|<X$ ), and

$$
\left\{\begin{array}{l}
\zeta(r, t)=\frac{1}{X+Y \cos \omega t}+\alpha\left(Y^{2}-X^{2}\right) \frac{r^{2}}{(X+Y \cos \omega t)^{2}} \\
u(r, t)=-\frac{Y \omega \sin \omega t}{X+Y \cos \omega t}\left(\frac{x}{2}, \frac{y}{2}\right)^{t}
\end{array}\right.
$$

The solution is periodic with a period $\tau=\frac{2 \pi}{\omega}$. The computational domain $\Omega$ is a square of length $L=8000 \mathrm{~m}$ centered at the origin. We set $\alpha=1.610^{-7} \mathrm{~m}^{-1}, X=1 \mathrm{~m}^{-1}$, and $Y=-0.41884 m^{-1}$. We use for this test case (with no relevant boundaries) a structured triangular mesh. The threshold $\varepsilon$ is set to $10^{-6}$. We observe that the scale of this test case is close to realistic applications, the order of magnitude of the water depth being around $2 \mathrm{~m}$ on a domain of kilometric size.

The solution is illustrated at different times in Figure 13. It was obtained with $p=1$ on a triangular mesh obtained by cutting rectangles of a $50 \times 50$ Cartesian mesh. The relative error in global mass conservation is less than $0.0002 \%$, confirming that the average of $\zeta_{h}$ almost never takes negative values. The $L^{2}$-norm of the error on the water depth is presented on Figure 14 . Two different behaviors appear. During the first half period $(t \in[0 ; \tau / 2])$, the water spreads and flooding occurs. For $p=0, p=1$, and $p=2$, the orders of convergence are respectively $0.9,1.4$, and 1.5. These results are close to expected orders of convergence (respectively, 0.5 , 1.5 , and 1.5). However, for the second half period $(t \in[\tau / 2 ; \tau])$, the water flows back and drying occurs. For $p=0$, the order of convergence is close to 0.5 , while for $p=1$ and $p=2$, the orders of convergence are close to each other and vary from around 1.1 down to 0.5. This means that the drying algorithm does not perform as well as expected. One can remark that the flooding and drying algorithm plays the role of a limiter. In the flooding zones, it has to limit numerical oscillations due to high order accuracy. However, in the drying zones, it has to limit both numerical oscillations and the physical drying process.

These two different behaviors can be illustrated by computing numerically the actual radius of the flooded zone during the computation. More precisely, we can compute (using the values of the discrete solution at quadrature points) the following radii: 


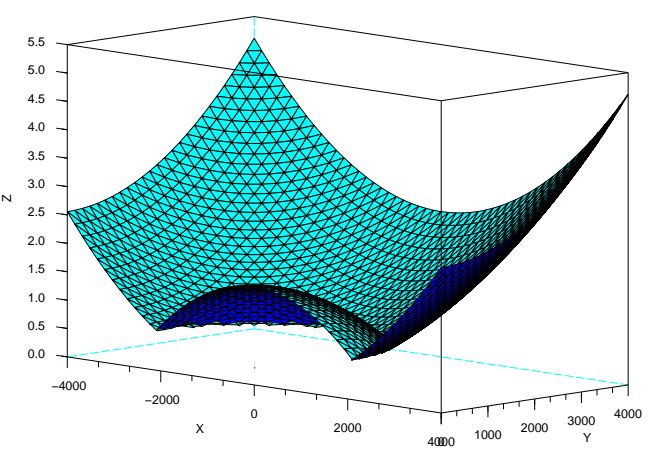

$$
t=0
$$

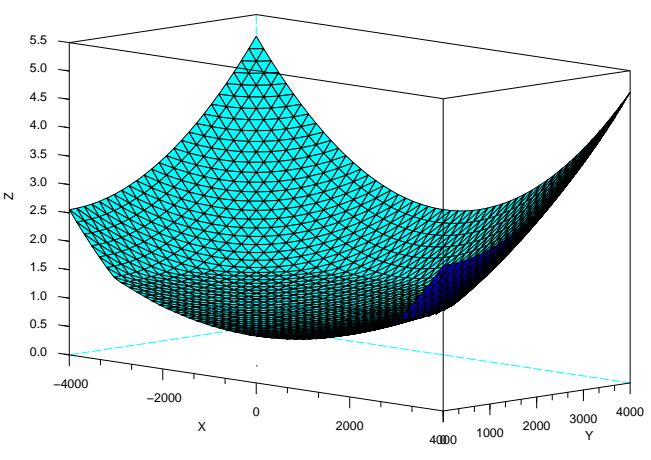

$$
t=\frac{2 \tau}{6}
$$

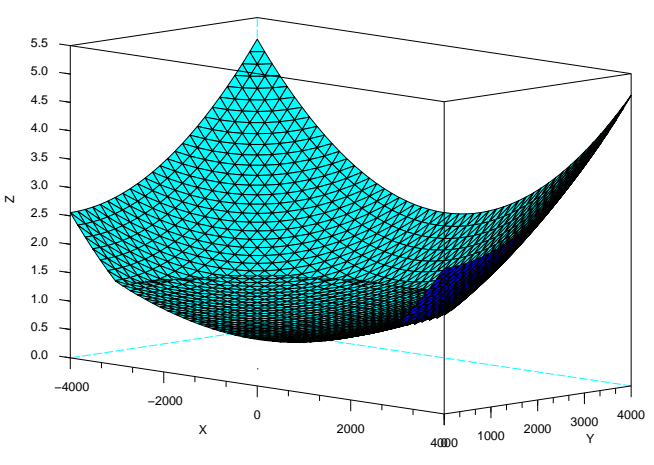

$$
t=\frac{4 \tau}{6}
$$

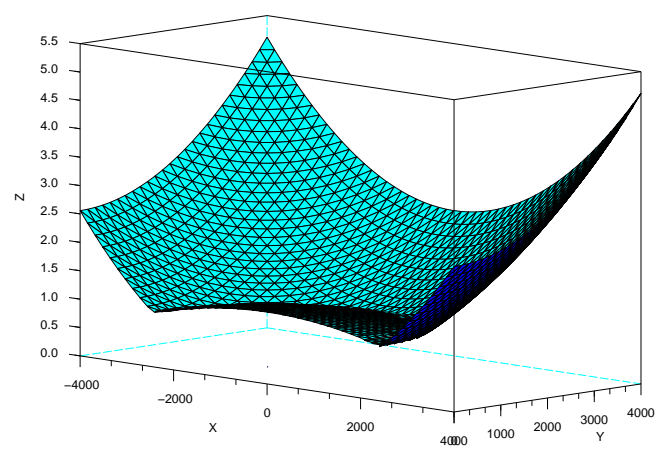

$$
t=\frac{\tau}{6}
$$

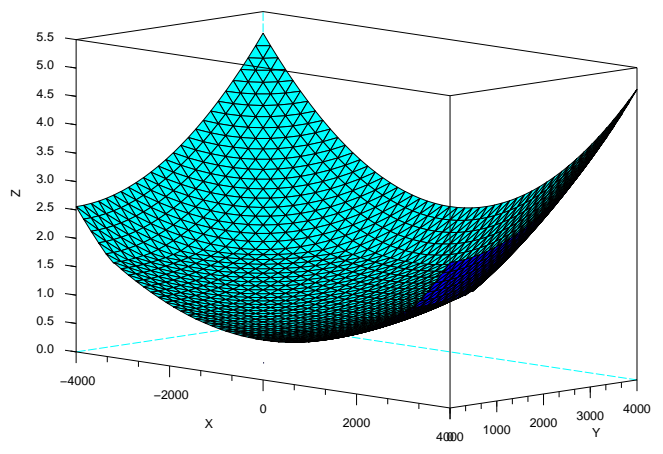

$$
t=\frac{3 \tau}{6}
$$

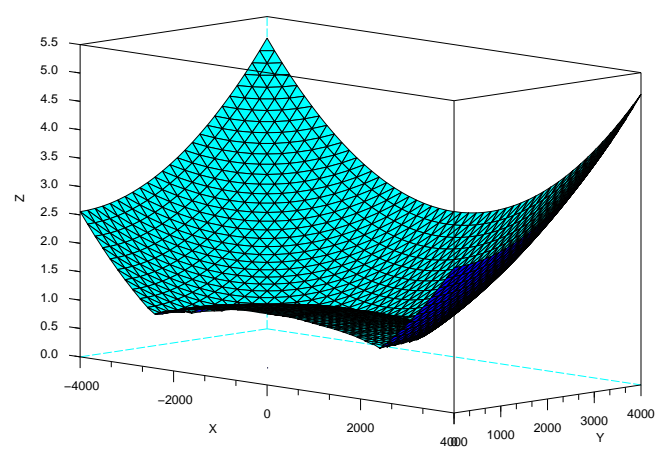

$t=\frac{5 \tau}{6}$

Figure 13. Parabolic bowl: approximate water depth for $p=1$ at times $t=i \frac{\tau}{6},(0 \leq i \leq 5)$. 

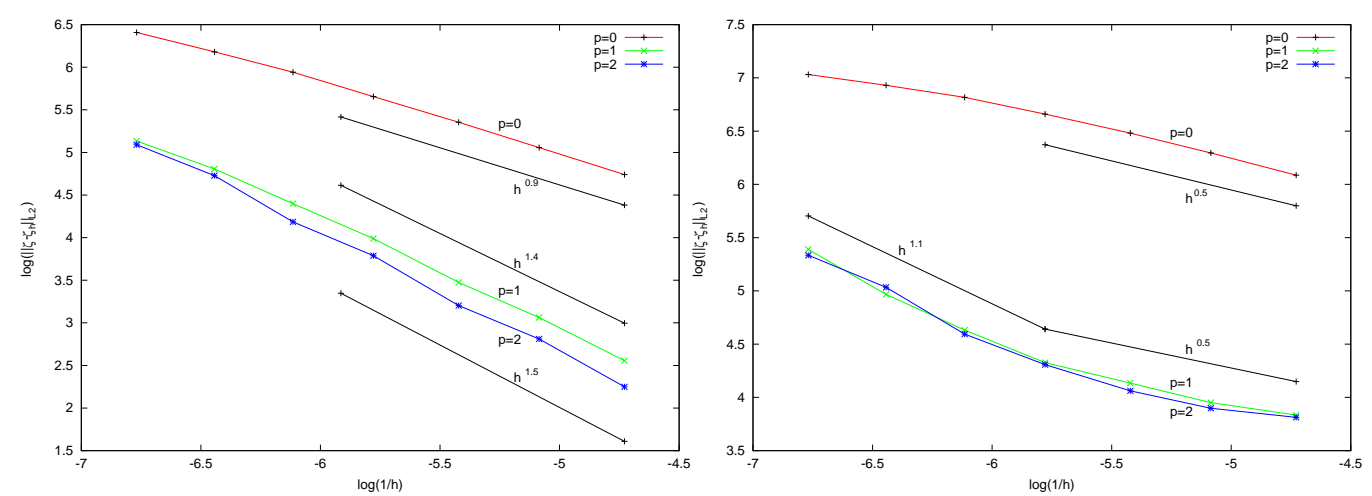

Figure 14. Parabolic bowl: $\max _{t \leq \frac{\tau}{2}}\left(\left\|\left(\zeta_{h}-\zeta\right)(t, .)\right\|_{L^{2}(\Omega)}\right)$ (left) and $\max _{t \leq \tau}\left(\left\|\left(\zeta_{h}-\zeta\right)(t, .)\right\|_{L^{2}(\Omega)}\right)$ (right) for $p \in\{0,1,2\}$.
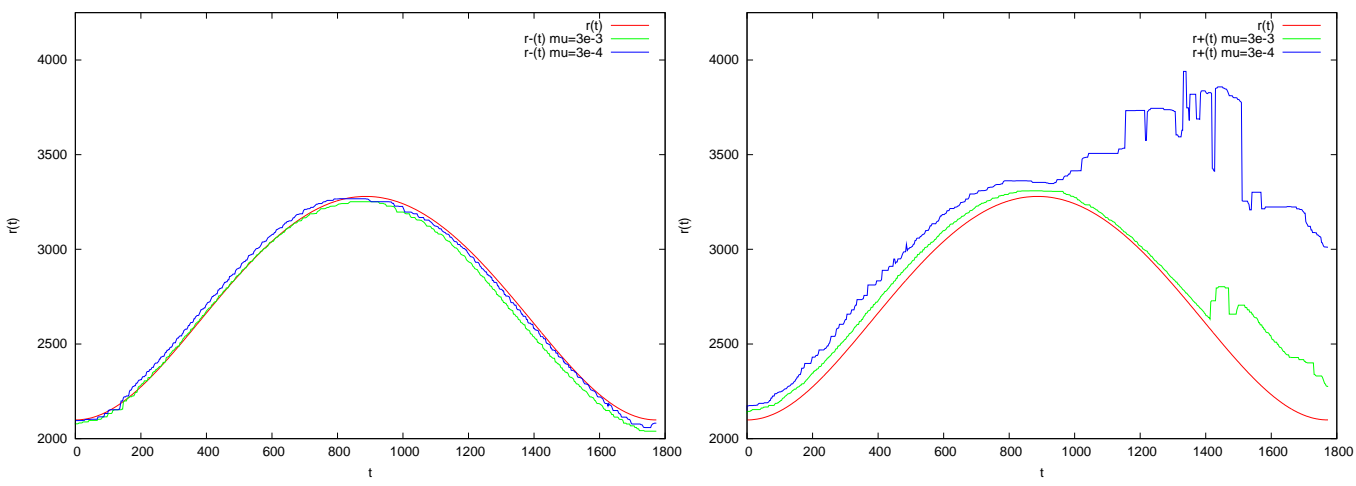

Figure 15. Parabolic bowl: $r^{-}(t, \mu)$ (left) and $r^{+}(t, \mu)$ (right) for $\mu \in\left\{10^{-2.5}, 10^{-3.5}\right\}$.

- the exact radius of the flooded zone $r(t)=\sqrt{\frac{X+Y \cos \omega t}{\alpha\left(X^{2}-Y^{2}\right)}}$;

- for any threshold $\mu, r^{-}(t, \mu)=\min _{\{(x, y) / \zeta(t, x, y) \leq \mu\}}\left(\sqrt{x^{2}+y^{2}}\right)$;

- for any threshold $\mu, r^{+}(t, \mu)=\max _{\{(x, y) / \zeta(t, x, y)>\mu\}}\left(\sqrt{x^{2}+y^{2}}\right)$.

By definition, $r^{-}(t, \mu) \leq r^{+}(t, \mu)$ with equality for all $\mu$ if $\zeta$ is monotonically decreasing. Discrepancies between $r^{-}(t, \mu)$ and $r^{+}(t, \mu)$ indicate that $\zeta$ oscillates around the threshold $\mu$. Furthermore, as $\mu \rightarrow 0, r^{+}(t, \mu)$ and $r^{-}(t, \mu)$ should be close to $r(t)$. In the zone $r<r^{-}(t, \mu)$, the ground can be considered as flooded (since $\zeta(t, x, y)>\mu$ ). On the contrary, in the zone $r>r^{+}(t, \mu)$, the ground can be considered as dry (since $\left.\zeta(t, x, y)<\mu\right)$. The zone $r^{-}(t, \mu) \leq r \leq r^{+}(t, \mu)$ is where the ground is marginally flooded. The different curves for $\mu \in\left\{10^{-2.5}, 10^{-3.5}\right\}$ are plotted on Figure 15 (polynomial order $p=1$, triangular mesh obtained by cutting rectangles of a $100 \times 100$ Cartesian mesh). The left part of Figure 15 shows 
that the flooded zone is quite accurately captured. The right part of the figure shows that the dry zone is not accurately captured during the drying phase (areas with small $\zeta$ are actually expanding during the computation for $\left.\mu=10^{-3.5}\right)$. In particular, observing $r^{+}\left(t, \mu=10^{-3.5}\right)$ yields a possible explanation of accuracy loss in the drying phase of the computation: while zones with $\zeta>10^{-3.5}$ remain limited during the flooding phase, they spread (or at least do not diminish) during the drying phase, where large areas with small $\zeta$ remain. Additional investigations on that specific behavior are under way. Anyway, one should keep in mind that these considerations are aimed at obtaining the sharpest possible asymptotic behavior for the numerical method, while spurious water heights below one millimeter are not a concern in practical simulations.

\section{Conclusions}

In this work, we have designed a well-balanced RKDG scheme for the shallow-water equations. In the absence of drying processes, the scheme performs well on structured or unstructured, fitted or unfitted meshes. As with classical CFE methods, the scheme delivers accurate solutions with high-order convergence rates whenever the solution is smooth enough. At the same time, the scheme can handle various nonsmooth wave structures (shocks, rarefaction fans), as FV methods. For drying processes, the scheme behaves satisfactorily in the present test case, since spurious oscillations where the water depth takes small values can be controlled below one millimeter over a domain with kilometric scale.

\section{Appendix. The HLLE and HLLC fluxes}

Let $K \in \mathcal{T}_{h}$, let $\sigma \in E_{h}^{i}(K)$ and let $K_{\sigma}$ be the element of $\mathcal{T}_{h}$ sharing the interface $\sigma$ with $K$. Let $x_{\sigma}$ be an integration point on $\sigma$. Let $W_{K}=\left(\zeta_{K}, u_{K} \zeta_{K}\right)$ and $W_{K_{\sigma}}=\left(\zeta_{K_{\sigma}}, u_{K_{\sigma}} \zeta_{K_{\sigma}}\right)$ be the two states on both sides of $x_{\sigma}$. Recall that $n_{K, \sigma}$ denotes the unit outward normal of $K$ on $\sigma$.

The HLLE flux is used in one space dimension. This numerical flux is based on the approximation that the solution consists of three states, namely $W_{K}, W_{\sigma}$ and $W_{K_{\sigma}}$, separated by two waves of speeds $c_{\sigma}^{ \pm}$. Letting $v_{K}=u_{K} \cdot n_{K, \sigma}$ and $v_{K_{\sigma}}=u_{K_{\sigma}} \cdot n_{K, \sigma}$, the wave speeds are evaluated as

$$
\begin{aligned}
& c_{\sigma}^{+}:=\max \left(0, \max \left(v_{K_{\sigma}}+\sqrt{g \zeta_{K_{\sigma}}}, v_{\sigma}^{*}+\sqrt{g \zeta_{\sigma}^{*}}\right)\right), \\
& c_{\sigma}^{-}:=\min \left(0, \min \left(v_{K}-\sqrt{g \zeta_{K}}, v_{\sigma}^{*}-\sqrt{g \zeta_{\sigma}^{*}}\right)\right),
\end{aligned}
$$

where

$$
\zeta_{\sigma}^{*}:=\frac{\zeta_{K}+\zeta_{K_{\sigma}}}{2}, \quad v_{\sigma}^{*}:=\frac{\sqrt{\zeta_{K}} v_{K}+\sqrt{\zeta_{K_{\sigma}}} v_{K_{\sigma}}}{\sqrt{\zeta_{K}}+\sqrt{\zeta_{K_{\sigma}}}},
$$

are the so-called Roe-averaged values. Then, the HLLE flux is evaluated as

$$
\phi_{*}^{\mathrm{HLLE}}\left(W_{K}, W_{K_{\sigma}}, n_{K, \sigma}\right):=\frac{1}{2}\left(\mathbb{F}_{1}\left(W_{K}\right)+\mathbb{F}_{1}\left(W_{K_{\sigma}}\right)\right) n_{K, \sigma}+\frac{1}{2} Q_{\sigma}\left(W_{K}-W_{K_{\sigma}}\right),
$$

with

$$
Q_{\sigma}:=\frac{c_{\sigma}^{+}+c_{\sigma}^{-}}{c_{\sigma}^{+}-c_{\sigma}^{-}}\left(\begin{array}{cc}
0 & 1 \\
-\left(v_{\sigma}^{*}\right)^{2}+g \zeta_{\sigma}^{*} & 2 v_{\sigma}^{*}
\end{array}\right)-2 \frac{c_{\sigma}^{+} c_{\sigma}^{-}}{c_{\sigma}^{+}-c_{\sigma}^{-}} I_{2}
$$


where $I_{2}$ is the identity matrix in $\mathbb{R}^{2,2}$.

In two space dimensions, the HLLC flux is preferred to the HLLE flux since the latter suffers from difficulties in resolving contact discontinuities and tangential waves. The HLLC flux is based on the approximation that the solution consists of four states, namely $W_{K}, W_{\sigma}^{-}, W_{\sigma}^{+}$ and $W_{K_{\sigma}}$, separated by three waves of speeds $c_{\sigma}^{ \pm}$and $c_{\sigma}$. The wave speeds are evaluated as

$$
\begin{aligned}
c_{\sigma}^{-} & :=\min \left(v_{K}-\sqrt{g \zeta_{K}}, v_{K_{\sigma}}-\sqrt{g \zeta_{K_{\sigma}}}\right), \\
c_{\sigma}^{+} & :=\min \left(v_{K}+\sqrt{g \zeta_{K}}, v_{K_{\sigma}}+\sqrt{g \zeta_{K_{\sigma}}}\right) \\
c_{\sigma} & :=\frac{\frac{1}{2} g \zeta_{K}^{2}-\frac{1}{2} g \zeta_{K_{\sigma}}^{2}+\zeta_{K_{\sigma}} v_{K_{\sigma}}\left(c_{\sigma}^{+}-v_{K_{\sigma}}\right)-\zeta_{K} v_{K}\left(c_{\sigma}^{-}-v_{K}\right)}{\zeta_{K_{\sigma}}\left(c_{\sigma}^{+}-v_{K_{\sigma}}\right)-\zeta_{K}\left(c_{\sigma}^{-}-v_{K}\right)} .
\end{aligned}
$$

Then, the HLLC is evaluated as

$$
\begin{aligned}
\phi_{*}^{\mathrm{HLLC}}\left(W_{K}, W_{K_{\sigma}}, n_{K, \sigma}\right) & :=\frac{1}{2}\left(\mathbb{F}_{i}\left(W_{K}\right)+\mathbb{F}_{i}\left(W_{K_{\sigma}}\right)\right) n_{K, \sigma, i} \\
& +\frac{1}{2}\left(\left(\left|c_{\sigma}^{-}\right|-\left|c_{\sigma}\right|\right) W_{\sigma}^{-}+\left(\left|c_{\sigma}^{+}\right|-\left|c_{\sigma}\right|\right) W_{\sigma}^{+}+\left|c_{\sigma}^{-}\right| W_{K}+\left|c_{\sigma}^{+}\right| W_{K_{\sigma}}\right),
\end{aligned}
$$

with

$$
\frac{c_{\sigma}^{-}-c_{\sigma}}{c_{\sigma}^{-}-v_{K}} W_{\sigma}^{-}:=W_{K}+\left(\begin{array}{c}
0 \\
\zeta_{K}\left(c_{\sigma}-v_{K}\right) n_{K}
\end{array}\right),
$$

and

$$
\frac{c_{\sigma}^{+}-c_{\sigma}}{c_{\sigma}^{+}-v_{K_{\sigma}}} W_{\sigma}^{+}:=W_{K_{\sigma}}+\left(\begin{array}{c}
0 \\
\zeta_{K_{\sigma}}\left(c_{\sigma}-v_{K_{\sigma}}\right) n_{K}
\end{array}\right)
$$

\section{ACKNOWLEDGEMENT}

This work was partly supported by the Direction de la Recherche et des Affaires Scientifiques et Techniques (DRAST) of the Ministère de l'équipement, des transports, de l'aménagement, du tourisme et de la mer. Fruitful discussions with Ph. Sergent and V. Laborie (CETMEF, France) are gratefully acknowledged.

\section{REFERENCES}

1. A. J. C. Saint-Venant, Théorie du mouvement non-permanent des eaux avec application aux crues des rivières et à l'introduction des marées dans leur lit, C. R. Acad. Sci. Paris 73 (1871) 147-154.

2. J. J. Stoker, Water Waves : The Mathematical Theory with Applications, Interscience publishers, New York, 1957.

3. C. B. Vreugdenhil, Numerical Methods for Shallow-Water Flow, Kluwer Academic, Dordrecht, The Netherlands, 1994.

4. J. M. Hervouet, Hydrodynamique des écoulements à surface libre, modélisation numérique avec la méthode des éléments finis, Presses de l'École Nationale des Ponts et Chaussées, 2003.

5. T. Katsaounis, C. Makridakis, Relaxation models and finite element schemes for the shallow water equations, in: Hyperbolic problems: theory, numerics, applications, Springer, Berlin, 2003, pp. 621-631.

6. O. C. Zienkiewicz, P. Ortiz, A split-characteristic based finite element model for the shallow water equations, Int. J. Numer. Methods Fluids 20 (1995) 1061-1080.

7. E. Audusse, Modélisation hyperbolique et analyse numérique pour les écoulements en eaux peu profondes, Ph.D. thesis, Université de Paris VI, Paris (France) (2004). 
8. A. Bermúdez, M. E. Vázquez, Upwind methods for hyperbolic conservation laws with source terms, Computer and Fluids 23 (1994) 1049-1071.

9. T. Galloüet, J. M. Hérard, N. Seguin, Some approximate Godunov schemes to compute shallow-water equations with topography, Computer and Fluids 32 (2003) 479-513.

10. D. L. George, Numerical approximation of the nonlinear shallow-water equations with topography and dry beds, Ph.D. thesis, University of Washington, Washington (United States) (2004).

11. P. Lesaint, P.-A. Raviart, On a finite element method for solving the neutron transport equation, in: Mathematical aspects of finite elements in partial differential equations (Proc. Sympos., Math. Res. Center, Univ. Wisconsin, Madison, Wis., 1974), Math. Res. Center, Univ. of Wisconsin-Madison, Academic Press, New York, 1974, pp. 89-123. Publication No. 33.

12. W. Reed, T. Hill, Triangular mesh methods for the neutron transport equation, Tech. Rep. LA-UR-73-479, Los Alamos, NM, Los Alamos Scientific Laboratory (1973).

13. B. Cockburn, C. W. Shu, Runge-Kutta Discontinuous Galerkin methods for convection-dominated problems, J. Sci. Comput. 16 (3) (2001) 173-261.

14. B. Cockburn, Discontinuous Galerkin methods for convection-dominated problems, in: T. Barth, H. Deconinck (Eds.), High-order methods for computational physics, Vol. 9 of Lect. Notes Comput. Sci. Eng., Springer, Berlin, 1999, pp. 69-224.

15. V. Aizinger, C. Dawson, A discontinuous Galerkin method for two-dimensional flow and transport in shallow water, Adv. Water Resour. 25 (2002) 67-84.

16. V. R. Ambati, O. Bokhove, Space-time discontinuous Galerkin finite element method for shallow water flows, J. Comput. Appl. Math.To appear.

17. O. Bokhove, Flooding and drying in Discontinuous Galerkin Finite-Element discretizations of shallowwater equations. part I : One dimension, J. Comput. Phys. 22 (2004) 47-82.

18. C. Dawson, V. Aizinger, A discontinuous Galerkin method for three-dimensional shallow water equations, J. Sci. Comput. 22/23 (2005) 245-267.

19. C. Dawson, J. Proft, Coupled discontinuous and continuous Galerkin finite element methods for the depthintegrated shallow water equations, Comput. Methods Appl. Mech. Engrg. 193 (3-5) (2004) $289-318$.

20. C. Eskilsson, S. J. Sherwin, A triangular spectral/hp Discontinuous Galerkin method for modelling $2 \mathrm{~d}$ shallow water equations, Int. J. Numer. Methods Fluids 45 (6) (2004) 605-623.

21. F. X. Giraldo, J. S. Hesthaven, T. Warburton, Nodal high-order discontinuous Galerkin methods for the spherical shallow water equations, J. Comput. Phys. 181 (2) (2002) 499-525.

22. H. Li, R. Liu, The discontinuous Galerkin finite element method for the 2D shallow water equations, Math. Comput. Simulation 56 (3) (2001) 223-233.

23. J. F. Remacle, S. S. Frazão, X. Li, M. S. Shephard, Adaptive Discontinuous Galerkin Method for the Shallow Water Equations, Int. J. Numer. Methods FluidsTo appear.

24. D. Schwanenberg, J. Köngeter, A discontinuous Galerkin method for the shallow water equations with source terms, in: Discontinuous Galerkin methods (Newport, RI, 1999), Vol. 11 of Lect. Notes Comput. Sci. Eng., Springer, Berlin, 2000, pp. 419-424.

25. P. A. Tassi, O. Bokhove, C. A. Vionnet, Space discontinuous Galerkin method for shallow water flowskinetic and HLLC flux, and potential vorticity generation, Adv. Water Res. 30 (4) (2007) 998-1015.

26. B. Einfeldt, On Godunov-type methods for gas dynamics, SIAM J. Numer. Anal. 25 (2) (1988) $294-318$.

27. B. Einfeldt, C. D. Munz, P. L. Roe, B. Sjögreen, On Godunov-type methods near low densities, J. Comput. Phys. 92 (1991) 273-295.

28. M. O. Bristeau, B. Coussin, Boundary conditions for the shallow-water equations solved by kinetic schemes, Tech. Rep. RR-4282, INRIA (2001).

29. S. Piperno, Symplectic local time-stepping in non-dissipative DGTD methods applied to wave propagation problems, M2AN Math. Model. Numer. Anal. 40 (5) (2006) 815-841.

30. L. Krivodonova, J. Xin, J. F. Remacle, N. Chevaugeon, J. E. Flaherty, Shock detection and limiting with discontinuous Galerkin methods for hyperbolic conservation laws, J. Comput. Phys. 48 (2004) 323-338.

31. S. Noelle, N. Pankratz, G. Puppo, J. R. Natvig, Well-balanced finite volume schemes of arbitrary order of accuracy for shallow water flows, J. Comput. Phys. 213 (2006) 474-499.

32. F. Alcrudo, P. Garcia-Navarro, A high-resolution Godunov-type scheme in finite volumes for the $2 \mathrm{~d}$ shallowwater equations, Int. J. Numer. Methods Fluids 16 (1993) 489-505.

33. N. Goutal, F. Maurel, Proceeding of the second workshop on dam-break simulation, Tech. Rep. HE43/97/016/B, EDF (1997).

34. A. Ritter, Die Fortpflanzung der Wasserwellen, A. Verdeut. Ing. 36.

35. W. C. Thacker, Some exact solutions to the nonlinear shallow-water wave equations, J. Fluid Mech. 107 (1981) 499-508. 